\title{
Impact of selenium on mood and quality of life: a randomized controlled trial.
}

Margaret Rayman, Alexander Thompson, Margaret Warren-Perry, Rachel Galassini, Janet Catterick, Emma Hall, David Lawrence, Judith Bliss

School of Biomedical and Molecular Sciences, University of Surrey, Guildford, Great Britain, GU2 7XH

Margaret Rayman (Principal Investigator)

Alexander Thompson (Data Manager)

Margaret Warren-Perry (Data Manager)

Rachel Galassini (Study Manager)

Janet Catterick (Study Manager)

Clinical Trials \& Statistics Unit, Section of Clinical Trials, Institute of Cancer Research, Sutton, Great Britain, SM2 $5 N G$

Emma Hall (Statistician)

David Lawrence (Statistician)

Judith Bliss (Statistician)

Corresponding author Margaret Rayman, m.rayman@surrey.ac.uk

$$
\text { Phone: }+44(0) 1483686447 \text { Fax: }+44(0) 1483686481
$$

Text word count: 3225 Abstract word count: $286 \quad 3$ figures, 3 tables

Running title: Impact of selenium on mood.

Funding: Funding for the UK pilot of the PRECISE Trial was provided by the Cancer Research Campaign (now Cancer Research UK). Pharma Nord (Vejle, Denmark) provided the tablets for the study without charge. Wassen International Ltd (Leatherhead, UK) paid for the extra costs associated with the mood study. 


\begin{abstract}
Background: Selenium is known to be important to the brain, retaining it even in deficiency. To date, three small, published studies have suggested an effect of selenium supplementation or deprivation on mood in healthy volunteers. We investigated these findings on a much larger scale.
\end{abstract}

Methods: In this double-blind, placebo-controlled, multi-centre intervention trial, 501 UK subjects aged $60-74$ were randomly allocated to receive 100,200 or $300 \mu$ g selenium per day as high-selenium yeast or placebo yeast. Main outcome measures were: mood (POMS-BI questionnaire), "quality of life" (SF-36 questionnaire) and plasma selenium (ICP-MS). Each was measured at baseline and six months as planned in the protocol.

Results: Supplementation significantly increased plasma selenium concentrations above baseline values: from an overall mean (SD) of 90 (19) ng/g to 91 (26), 144 (27), 191 (41) and

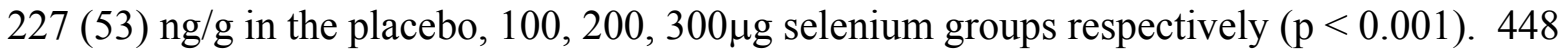
participants completed the POMS-BI questionnaires at both time points, with no significant differences in total mood or mood-subscale scores seen between the treatment doses. After six months of supplementation, mean (SD) total mood scores for the four tablet doses were 163 (36), $161(37), 162(33), 162(34), F_{3,443}=0.25, p=0.86$. Quality of life was similarly unaffected by supplementation.

Conclusions: Selenium supplementation did not appear to benefit mood or quality of life in these elderly volunteers. Though this is at odds with some previous results, our robust study design, much larger sample size and longer supplementation period, together with the evidence that the brain is a privileged site for selenium retention, suggest that this is a reliable 
finding. We therefore find no justification for increasing selenium intake with the aim of improving mood in this population.

Key words: selenium, mood, quality of life, elderly, POMS-BI, SF-36 
The assertion that selenium enhances mood is now commonly encountered both in the scientific (Rayman 2000) and in the popular press, yet is based on the results of only a few, small studies. We therefore took the opportunity to test this interesting hypothesis while conducting a pilot study for a much larger randomized trial that supplemented participants with selenium.

There are a number of indications that selenium is important to the brain: during selenium depletion the brain receives a priority supply (Behne et al 1988; Buckman et al 1993; Whanger 2001); neurotransmitter turnover rate is altered in selenium deficiency (Castano et al 1997); selenium supplementation can reduce intractable epileptic seizures (Weber et al 1991, Ramaekers 1994); low plasma selenium levels in the elderly have been associated with senility and accelerated cognitive decline (Berr et al 2000); Alzheimer's patients have lower brain selenium concentrations than controls (Corrigan et al 1991); sodium selenite protected against dopamine loss in a rat model of Parkinson's disease (Zafar et al 2003); a selenoenzyme (GPx1) protected against neuron loss in a mouse stroke model(Crack et al 2001). Furthermore, as the brain is deficient in catalase (Halliwell and Gutteridge 1985), peroxidation products such as $\mathrm{H}_{2} \mathrm{O}_{2}$ and lipid peroxides must be removed by the antioxidant selenoenzymes.

With respect to mood, two published studies have shown a beneficial effect of selenium as measured by the Profile of Moods States - Bipolar Form (POMS-BI) questionnaire (Lorr and McNair 1984). In a US study where 30 selenium-replete men were fed either a low $(32.6 \mu \mathrm{g} / \mathrm{d})$ or high $(226.5 \mu \mathrm{g} / \mathrm{d})$ selenium diet for 15 weeks, the mood of those on the low selenium diet significantly worsened while that of those on the high selenium diet significantly improved (Finley and Penland 1998). Unfortunately it is not clear whether diet type was allocated randomly. A similar finding was obtained in a double-blind

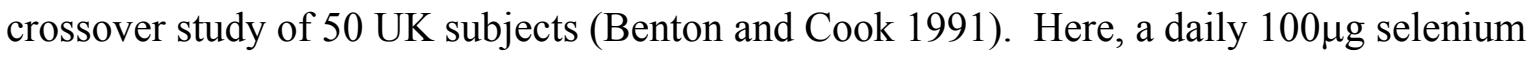


supplement as high-selenium yeast significantly decreased anxiety, depression and tiredness, with the effect being most marked in subjects consuming lesser amounts of dietary selenium (estimated by a food-frequency questionnaire). However, a further US study involving 11 men of adequate selenium status randomized to high $(356 \mu \mathrm{g} / \mathrm{d})$ or low $(13 \mu \mathrm{g} / \mathrm{d})$ selenium diets for 99 days failed to show any change in mood (Hawkes and Hornbostel 1996). That said, in subjects fed the low-selenium diet, the lower their initial selenium status the more their mood tended towards depression and hostility.

A subsequent randomized, double-blind US study that supplemented 115 HIVpositive drug abusers with $200 \mu \mathrm{g}$ selenium daily found no difference compared to placebo with respect to Total Distress scores (POMS) or depression (Beck Depression Inventory) (Shor-Posner et al. 2003). Higher plasma selenium levels were, however, associated with less anxiety (State-Trait anxiety questionnaire) and improved vigor (POMS). However, the authors comment that these improvements may have been confounded by the known beneficial effect of selenium on immune function, resulting in fewer mycobacterial infections (Shor-Posner et al 2003).

In the light of these reports, the effect of different doses of selenium on mood was compared to placebo as part of the UK PRECISE (PREvention of Cancer by Intervention with SElenium) Pilot Study.

\section{Materials and Methods}

\section{Participants and recruitment}

The UK PRECISE pilot study was a double-blind, placebo-controlled, randomized trial involving volunteers from four general practices affiliated to the Medical Research Council General Practice Research Framework (GPRF). Practices were deliberately chosen from areas of England with differing demographic characteristics: Guisborough and Linthorpe 
(NorthEast), Bromsgrove (West Midlands) and Bungay (East Anglia). Research nurses recruited similar numbers of men and women from each of three age groups: 60-64, 65-69 and 70-74 years. Exclusion criteria were: i) a Southwest Oncology Group performance status score $>1$ (i.e. incapable of carrying out light housework or office work); ii) active liver or kidney disease; iii) prior diagnosis of cancer (excluding non-melanoma skin cancer); iv) diagnosed HIV infection; v) on immunosuppressive therapy; vi) diminished mental capacity; vii) taking $\geq 50 \mu \mathrm{g} /$ day of selenium supplements in the previous six months (by patient report). The study had ethics committee approval and participants provided written informed consent to participate.

\section{Protocol}

Following a four-week placebo run-in, participants were randomly assigned to one of four treatment regimes: placebo, 100, 200 or $300 \mu \mathrm{g}$ of selenium per day for two years. The intervention agent was high-selenium yeast, SelenoPrecise ${ }^{\mathrm{TM}}$ (Pharma Nord, Vejle, Denmark) or an identical placebo yeast. Randomization was stratified by GP practice, gender and age group and used computer-generated permuted blocks. Research nurses telephoned the independent randomization service at the Clinical Trials and Statistics Unit at the Institute of Cancer Research (Sutton, UK) to obtain an anonymous code for each volunteer, and then gave the volunteer their corresponding pre-coded tablets. Participants and general practice personnel were blinded to treatment for as long as the participants remained on treatment. Demographic data, medical history, and other health-related information (including medication use) were collected at baseline. Participants completed mood and quality-of-life questionnaires and provided a blood sample at both baseline and six months (when visiting the practices for the purpose of the PRECISE pilot). The participants completed the questionnaires before blood was taken. Compliance with randomized treatment was 
determined by pill count, with participants considered compliant if they took at least $80 \%$ of their allocated tablets. Reasons for participant withdrawal were noted.

\section{Mood and Quality of Life assessment (outcome)}

Mood was assessed using the POMS-BI questionnaire (MHS, Toronto - formerly EDITS, San Diego; Lorr and McNair 1984). This questionnaire, validated in the US, contains 72 adjectives rated on a scale from "much like this" to "much unlike this" according to how the participants had been feeling in the past week. For this UK study, the adjective "mad" (meaning "angry" in US usage) was changed to "furious" and "full of pep" to "full of energy" with permission from EDITS. The adjectives are divided into six subscales, each scoring a maximum of 36 , and can be summed to provide a "Total mood score". Higher scores are associated with better mood. An independent researcher performed scoring checks to verify that original scores were accurate.

The Short Form 36 (SF-36) Health Survey (QualityMetric, Inc., Lincoln, USA) a standardized "quality-of-life" questionnaire, validated in Great Britain (Brazier et al. 1992; Garratt et al. 1993) that measures mental and physical well-being was also administered. The SF-36 gives scores for nine health concepts. Raw scores were transformed on a 0 to 100 scale (Medical Outcomes Trust 1994) with higher scores associated with a better quality of life.

\section{Se measurement (outcome)}

Lithium-heparin plasma was prepared in the practices from whole blood samples, stored at $80^{\circ} \mathrm{C}$ and sent to Central Science Laboratory, Sand Hutton, for selenium analysis by hydridegeneration Inductively-Coupled-Plasma Mass Spectrometry. Weighed plasma samples were prepared by microwave digestion (Multiwave, Perkin-Elmer, Bucks, UK) and reduced to 
selenium (IV), before being made up to volume for analysis. All reagents were "Analar" grade (or better) and the water used was Millipore-grade (18 M 2 ). Quality-control procedures were accredited under the UK Accreditation Scheme (UKAS). Accuracy was assured by analysis of certified reference materials namely: Seronorm Serum, mean value (10 determinations) 85.5ng/g, RSD 12.7\% (certified 86ng/g); NIST 1598 Bovine Serum, mean value (16 determinations) 43.5ng/g, RSD 6.2\% (certified 42.4 $\pm 3.5 \mathrm{ng} / \mathrm{g}$ ). The Limit of Detection was 5ng/g and the Mean Recovery 108\% (12 determinations). Plasma selenium measurements can be converted from $\mathrm{ng} / \mathrm{g}$ to $\mu \mathrm{g} / \mathrm{L}$ by multiplying by 1.027 , the density of plasma.

\section{Statistical analysis}

Factors affecting total mood score after six months of supplementation (the outcome) were determined by ANCOVA with baseline mood as the covariate. Randomized intervention was included as a four-level factor. Participants' age group, gender and practice location (the stratification factors) and two a priori defined factors of smoking status (smoker/given up/never smoked) and antidepressant medication (yes/no) were also considered. Factors significantly associated with six-month total mood score were included in the final multivariate model with randomized intervention. The assumptions of normality, homoskedasticity, linearity and homogeneity of regression slope were tested and met for each ANCOVA. All analyses were performed on the raw data, apart from the three stratification ANCOVAs and the one-way ANCOVAs by tablet type (active vs. placebo), smoking habit and antidepressant use that were carried out using square-transformed data so that the assumptions could be met. Baseline comparisons between the randomized groups were made using t-tests and ANOVA. SF-36 data were analyzed using non-parametric methods in lieu of two-way ANOVA (Sokal and Rohlf 2001), with time (baseline or six months) and tablet dose 
included as two main effects and an interaction. Chi-square tests were used to analyze the frequency of withdrawals. Participants who did not complete questionnaires at both baseline and follow-up have been excluded (complete-case analysis), however all those who did were included regardless of their compliance with allocated treatment. Statistical analysis was performed using SPSS v11.5.1 for Windows (SPSS Inc. Chicago).

\section{Results}

\section{Participants}

501 participants were recruited between June 2000 and July 2001 (figure 1).

\section{Compliance with treatment}

453 of the 467 participants (97\%) who completed six months were compliant according to pill count. Non-protocol use of over-the-counter selenium (“drop-ins”) was assessed by inspection of the distribution of plasma selenium concentrations in the placebo group at six months. Four of the 107 participants taking placebo (3.7\%) had a selenium status more than two standard deviations above the mean: reasonably consistent with the $2.5 \%$ expected by chance (being approximately normally distributed). We have made the assumption therefore, that "drop-ins" were rare. Three participants reported using supplements containing $25 \mu \mathrm{g}$ of selenium: since this was within the $50 \mu \mathrm{g}$ daily limit imposed by our exclusion criteria, they have been included in the analysis.

\section{Withdrawals}

Thirty-four participants (7\%) withdrew from treatment within the first six months. There was no significant difference in treatment withdrawals between groups $(7,10$, and 12 in the 
placebo, 100, 200 and $300 \mu$ groups respectively: $\left.X^{2}=3.41, d f=3, p=0.33\right)$. Of these, 13 withdrew because of adverse events, seven of which were abdominal/stomach problems. Other reasons for discontinuation appeared unrelated to treatment (figure 1).

\section{Selenium status}

Blood was obtained successfully from 483 participants at baseline and 429 at six months (with 421 having assessment at both time points). At baseline, as expected, there were no significant differences in selenium concentration between the four treatment groups $\left(\mathrm{F}_{3,479}=0.70, \mathrm{p}=0.55\right)$. Overall mean (SD) for all participants was 90 (19) ng/g (i.e. 92 (20) $\mu \mathrm{g} / \mathrm{L})$. This was higher than the $79 \mu \mathrm{g} / \mathrm{L}$ found by the National Diet and Nutrition Survey (Bates et al. 2002). After six months of supplementation, plasma selenium rose significantly to $145(27), 191(41)$ and $227(53) \mathrm{ng} / \mathrm{g}$ in the 100, 200 and $300 \mu \mathrm{g} / \mathrm{d}$ treatment groups respectively $\left(\mathrm{F}_{3,416}=308, \mathrm{p}<0.001\right)$ : figure 2 . Selenium status did not change significantly in the placebo group $(\mathrm{t}=0.34, \mathrm{df}=102, \mathrm{p}=0.74)$.

\section{Mood (POMS-BI)}

The POMS-BI questionnaire was completed by 448 participants at both time points. Mean scores by intervention dose are shown in table 1. At baseline, total mood score did not differ significantly between the four treatment doses, the three age groups, or the four practice locations. However, baseline mood was significantly higher in men than women $(t=5.28$, $\mathrm{df}=446, \mathrm{p}<0.001$ ), being: 170 (30) for men versus 154 (33) for women, mean difference $=16$ (95\% CI: $10-22)$.

Following six months of supplementation with selenium, total mood score (adjusted for individual variation in baseline mood) was independent of dose $\left(F_{3,443}=0.25, p=0.86\right)$. 
Furthermore, none of the stratification factors (gender, age group, or practice location) significantly influenced adjusted six-month total mood scores (table 3). Similarly, when all participants receiving the active doses $(100,200,300 \mu \mathrm{g} / \mathrm{d})$ were pooled and compared to placebo, no effect of selenium supplementation per se was found $\left(\mathrm{F}_{1,445}=0.59, \mathrm{p}=0.44\right)$ : figure 2. No significant effect on mood was observed from taking antidepressant medication $(n=33$ participants; $\left.\mathrm{F}_{1,445}=0.9, \mathrm{p}=0.35\right)$ nor according to participants' smoking habits $\left(\mathrm{F}_{2,444}=1.1\right.$, $\mathrm{p}=0.33)$

Participants' baseline selenium status did not significantly affect the outcome of supplementation on mood. When tertiles of baseline selenium status were included in the analysis (cut points at 79.6 and $95.4 \mathrm{ng} / \mathrm{g}$ ), no significant differences in six-month total mood score were seen between the tertiles $\left(\mathrm{F}_{2,419}=1.62, \mathrm{p}=0.2\right)$, nor any interaction with dose $\left(\mathrm{F}_{6,419}=0.23, \mathrm{p}=0.97\right)$. Initial (i.e. baseline) $\operatorname{mood}$ was the only significant predictor of sixmonth mood in any of the models tested.

When analyzed using the level of selenium status attained after supplementation (by quintiles of selenium status) rather than by intervention dose (thus taking account of possible "drop-ins" and non-compliers), no significant difference in mood was found between the quintiles $\left(\mathrm{F}_{4,403}=0.49, \mathrm{p}=0.75\right)$. Finally, separate analyses of the six individual subscales that sum to create the total mood score failed to reveal any significant differences between treatment doses (data not reported).

\section{Quality of life (SF-36)}

The SF-36 was completed by 398 participants at both time points. As shown previously (Bowling et al. 1999) the health concept scores were non-normal in their distribution. No significant association was observed between any of the nine health concepts and tablet dose (table 2). 


\section{Discussion}

We set out to investigate whether we could confirm that selenium enhanced mood in a large, randomized, double-blind, multi-centre, placebo-controlled trial. Our study is by far the largest to date to investigate the effect of selenium on mood in healthy individuals: 448 versus 91 participants in total in the other studies. In agreement with Hawkes and Hornbostel (1996) but in contrast to Benton and Cook (1991) and Finley and Penland (1998), we found no evidence that additional selenium enhanced mood or any of its subscales despite significant increases in plasma selenium. In support of our findings with the POMS-BI questionnaire, we found no significant effect of selenium supplementation on any of the subscales of the SF-36 that relate to mental state.

Possible explanations for these different findings include: the greater age of our participants; our longer treatment period; better baseline selenium status and mood in our cohort than in some earlier work (Benton and Cook 1991) and an alternative interpretation of Finley and Penland's data (1998). These possibilities are addressed below.

Firstly the question of age: our participants were considerably older than in the studies of Benton and Cook (mean 36, range 14-74 years), Finley and Penland (range 18-45 years) and Hawkes and Hornbostel (mean 33, range 20-45 years). While we chose the POMS-BI questionnaire to allow direct comparison with these previous studies, unlike the SF-36, it has not been validated in the elderly. Nevertheless, equivalent coefficients of variation in scores for the two questionnaires suggest that responses to the POMS-BI were no more variable than to the SF-36. However, changes associated with ageing could explain the different results observed.

Our participants were treated for 26 weeks compared to five and 15 weeks (Benton and Cook 1991, Finlay and Penland 1998) in the other studies. Finlay and Penland (1998) 
showed plasma selenium to plateau after six weeks of dietary supplementation where the major selenium source was wheat. While wheat and selenium-yeast behave identically in supplementation studies in terms of their effect on plasma selenium (both having selenomethionine as their main selenium-containing species) (Levander et al. 1983), other studies suggest the process can take considerably longer: 11 weeks (Alfthan et al. 1991) and 25 weeks (Thomson et al. 1993). Furthermore, selenoenzyme activity can take from five to 20 weeks to plateau depending on the tissues involved (Thomson et al. 1993). This suggests that in a five-week study (Benton and Cook 1991), and perhaps to a lesser extent a 15-week study (Finlay and Penland 1998), selenium status and selenoenzyme activity may not have reached a steady state in all tissues. By contrast, after 26 weeks, our participants' selenium status and selenoenzyme activity had probably stabilized. This may explain some of the differences between studies, but we are unable to suggest a mechanism.

Mean baseline selenium status in our participants $(92 \mu \mathrm{g} / \mathrm{L})$ was higher than that found in free-living subjects $(79 \mu \mathrm{g} / \mathrm{L})$ in the UK National Diet and Nutrition Survey (Bates et al. 2002). This is perhaps not too surprising as our participants were younger (60-74 versus 6574) and were healthy volunteers, a quarter of whom came from East Anglia where selenium status is relatively high (Caygill et al 1989). While Benton and Cook (1991) did not measure selenium status, their subjects, recruited in South Wales, may have had a lower selenium status. Furthermore, mean baseline mood score in Benton and Cook's subjects was also lower than in both this study and that of Hawkes and Hornbostel (1996). It then increased to a level similar to that found at baseline and follow-up in this study and Hawkes and Hornbostel's work. However, if low selenium status were the cause of low mood score, one might have expected to see a significant interaction between selenium status and intervention dose. We found none. Moreover, it is not clear from the results reported by Benton and 
Cook (1991) that their analysis appropriately accounted for the crossover design of their study.

Lastly, it is possible that Finley and Penland (1998) may have been observing regression to the mean rather than changes in mood accompanying high- or low-selenium diets (Macallan and Sedgwick 2000). Their two treatment groups were not equivalent in mood at the beginning of the study, the low selenium group having higher baseline scores than the high selenium group. This initial difference in mood (illustrated in their paper) was greater than the change exhibited by either group during the trial. Furthermore, the low selenium diet did not significantly reduce plasma selenium levels from baseline values.

On reflection, it is perhaps unsurprising that we saw no effect of selenium supplementation on mood. Animal studies have shown that the brain is a privileged site for selenium retention in deficiency (Behne et al 1988; Buckman et al 1993; Whanger 2001). For example, Behne and colleagues (Behne and Kyriakopoulos 2001) showed that even when rats had been severely depleted of selenium through six generations resulting in a decrease in liver, skeletal muscle and blood selenium concentrations to below $1 \%$ of normal levels, the brain still contained $60 \%$ of the concentration found in controls.

Mean baseline plasma selenium in our cohort was arguably in the replete range (Alfthan et al. 1991; Thomson et al. 1993; Duffield et al.1999) where selenoproteins may already be adequately supplied with selenium. For example, the iodothyronine deiodinases, are adequately supplied with selenium at this plasma concentration (Beech et al 1995; Duffield et al 1999), so there is no risk of hypothyroidism due to selenium deficiency nor its associated adverse mood states of depression, lethargy and malaise (Reus 1993). This is not necessarily to imply that the selenium status of our participants was optimal, since it has been found that selenium at "supra-nutritional" intakes may confer protection against cancer and 
augment the immune response (Combs 2001; Kiremidjian-Schumacher et al. 1994; Rayman 2000).

In conclusion, we have found that selenium supplementation in a randomized, doubleblind, multi-centre, placebo-controlled trial in $501 \mathrm{UK}$ elderly subjects of moderate selenium status did not benefit their mood or quality of life. Though this is at odds with the findings of two smaller studies in other healthy individuals (Benton and Cook 1991, Finley and Penland 1998) our robust study design, large sample size and longer supplementation period, taken together with the evidence that the brain is a privileged site for selenium retention, suggest that this is a reliable finding. Populations of lower selenium status, worse mood, or younger age however, may show a different outcome. We find no justification for increasing selenium intake to improve mood in the general UK elderly population.

\section{Acknowledgements}

The authors would like to thank the personnel of the MRC General Practice Research Framework, in particular the research nurses, Anna Williams, Angela Ince, Anne Hall, Jill Wilkinson, Lesley Hand and Cynthia Dixon who recruited and followed up the participants. Thanks are also due to Drs. Marr (The Garth Surgery, Guisborough), Leci (Churchfields Surgery, Bromsgrove), Hand (Bungay Medical Practice, Bungay) and Robertson (Cambridge Medical Group, Linthorpe) and particularly to their patients who generously agreed to take part in the PRECISE Pilot Study. 


\section{References}

Alfthan G, Aro A, Arvilommi H \& Huttunen JK. Selenium metabolism and platelet glutathione peroxidase activity in healthy Finnish men: effect of selenium yeast, selenite, and selenate. Am J Clin Nutr1991; 53: 120-125.

Bates CJ, Thane CW, Prentice A, Delves HT. Selenium status and its correlates in a British National Diet and Nutrition Survey: people aged 65 years and over. J Trace Elem Med Biol $2002 ; 16: 1-8$.

Beech SG, Walker SW, Beckett GJ, Arthur JR, Nicol F, Lee D. Effect of selenium depletion on thyroidal type-1 deiodinase activity in isolated human thyrocytes and rat thyroid and liver. Analyst 1995; 120: 827-31.

Behne D, Hilmert H, Scheid S, Gessner H, Elger W. Evidence for specific selenium target tissues and new biologically important selenoproteins. Biochem Biophys Acta 1988; 966: 1221.

Behne D, Kyriakopoulos A. Mammalian selenium-containing proteins. Annu Rev Nutr 2001; 21: 453-73.

Benton D, Cook R. The impact of selenium supplementation on mood. Biol Psychiatry 1991; 29: 1092-1098.

Berr C, Balansard B, Arnaud J, Roussel AM, Alperovitch A. Cognitive decline is associated with systemic oxidative stress - the EVA study. J Am Geriatr Soc 2000; 48: 1285-1291. 
Bowling A, Bond M, Jenkinson C, Lamping DL. Short From 36 (SF-36) Health Survey questionnaire: which normative data should be used? Comparisons between the norms provided by the Omnibus Survey in Britain, the Health Survey for England and the Oxford Healthy Life Survey. J Public Health Med 1999; 21(3): 255-270.

Brazier JE, Harper R, Jones NM, O'Cathain A, Thomas KJ, Usherwood T, Westlake L. Validating the SF-36 health survey questionnaire: new outcome measure for primary care. BMJ 1992; 305: 160-164.

Buckman T, Sutphin MS, Eckhert CD. A comparison of the effects of dietary selenium on selenoprotein expression in rat brain and liver. Biochem Biophys Acta 1993; 1163: 176-184.

Castano A, Ayala A, Rodriguez-Gomez JA, Herrera AJ, Cano J, Machado A. Low selenium diet increases the dopamine turnover in prefrontal cortex of the rat. Neurochemistry International 1997; 30: 549-555.

Caygill CPJ, Lavery K, Judd PA, Hill MJ, Diplock AT. Selenium status and gastric cancer in two regions of Norfolk. Food Addit Contam 1989; 6: 359-363.

Combs GF Jr. Selenium in global food systems. Brit J Nutr 2001; 85: 517-547.

Corrigan FM, Reynolds GP, Ward NI. Reductions of zinc and selenium in brain in Alzheimer's disease. Trace Elem Med 1991; 8: 1-5. 
Crack PJ, Taylor JM, Flentjar NJ, de Haan J, Hertzog P, Ianello RC, Kola I. Increased infarct size and exacerbated apoptosis in the glutathione peroxidase-1 (Gpx-1) knockout mouse brain in response to ischemia/reperfusion injury. J Neurochem 2001; 78: 1389-99.

Duffield AJ, Thomson CD, Hill KE \& Williams S. An estimation of selenium requirements for New Zealanders. Am J Clin Nutr 1999; 70: 896-903.

Finley JW, Penland JG. Adequacy or deprivation of dietary selenium in healthy men: clinical and psychological findings. J Trace Elem Exp Med 1998; 11: 11-27.

Garratt AM, Ruta DA, Abdalla MI, Buckingham JK, Russell IT. The SF-36 health survey questionnaire: an outcome measure suitable for routine use within the NHS? BMJ 1993; 306: $1440-444$

Halliwell B, Gutteridge JMC. Oxygen radicals and the nervous system. TINS. 1985; 8: 2226.

Hawkes WC, Hornbostel L. Effects of dietary selenium on mood in healthy men living in a metabolic research unit. Biol Psychiatry 1996; 39: 121-128

Kiremidjian-Schumacher L, Roy M, Wishe HI, Cohen MW, Stotsky G. Supplementation with selenium and human immune cell functions. Biol Trace Elem Res 1994; 41: 115-127. 
Levander OA, Alfthan G, Arvilomni H, Gref CG, Huttunen JK, Kataja M et al.

Bioavailability of selenium to Finnish men as assessed by platelet glutathione peroxidase activity and other blood parameters. Am J Clin Nutr 1983; 37: 887-897.

Lorr M, McNair DM. Manual. Profile of Mood States. Bi-Polar Form (POMS-BI), EDITS, San Diego; 1984. p. 1-8.

Macallan DC, Sedgwick P. Selenium supplementation and selenoenzyme activity. Clin Sci 2000; 99: 579-81.

Medical Outcomes Trust. How to score the SF-36 Health Survey. PO Box 1917, Boston, MA $02205 ; 1994$.

Ramaekers VTh, Calomme M, Vanden Berghe D, Makropoulos W. Selenium deficiency triggering intractible seizures. Neuropediatrics 1994; 25: 217-223.

Rayman MP. The importance of selenium to human health. Lancet 2000; 356: 233-241.

Reus VI. Psychiatric aspects of thyroid disease In: Joffe RT, Levitt AJ, eds. Thyroid axis and Psychiatric illness. Ch 5, 171-194. Washington, DC: American Psychiatric Press, Inc. 1993.

Shor-Posner G, Lecusay R, Miguez MJ, Moreno-Black G, Zhang G, Rodriguez N, et al. Psychological burden in the era of HAART: Impact of selenium therapy. Intl J Psychiatry in Medicine 2003; 33 (1): 55-69. 
Sokal RR, Rohlf FJ. Biometry. $3^{\text {rd }}$ edition. New York: WH Freeman and Company, 2001.

Thomson CD, Robinson MF, Butler JA, Whanger PD. Long-term supplementation with selenate and selenomethionine: selenium and glutathione peroxidase (EC 1.11 1.9) in blood components of New Zealand women. Brit J Nutr 1993; 69: 577-588.

Weber GF, Maertens P, Meng X, Pippenger CE. Glutathione peroxidase deficiency and childhood seizures. Lancet 1991; 337: 1443-44.

Whanger PD. Selenium and the brain: a review. Nutr Neurosci 2001; 4: 81-97.

Zafar KS, Siddiqui A, Sayeed I, Ahmad M, Salim S, Islam F. Dose dependent protective effect of selenium in a rat model of Parkinson's disease: neurobehavioral and neurochemical evidences. J Neurochem 2003; 84: 438-46. 
Table 1. POMS-BI subscale and total mood scores at baseline and six months by tablet dose. Data are means (SD) scored out of 36 for each subscale and 216 for the composite total mood score. Higher scores indicate better mood.

\begin{tabular}{|c|c|c|c|c|c|}
\hline \multirow[b]{3}{*}{ Mood subscale } & \multirow[b]{3}{*}{ Month } & \multicolumn{4}{|c|}{ Intervention dose } \\
\hline & & Placebo & $100 \mu \mathrm{g}$ & $200 \mu \mathrm{g}$ & $300 \mu \mathrm{g}$ \\
\hline & & $\mathrm{n}=112$ & $\mathrm{n}=111$ & $\mathrm{n}=116$ & $\mathrm{n}=109$ \\
\hline \multirow[t]{2}{*}{ Composed - Anxious } & 0 & $27(8)$ & $27(7)$ & $28(7)$ & $27(7)$ \\
\hline & 6 & $27(7)$ & $27(8)$ & $27(7)$ & $28(6)$ \\
\hline \multirow[t]{2}{*}{ Agreeable - Hostile } & 0 & $30(5)$ & $30(6)$ & $30(6)$ & $30(5)$ \\
\hline & 6 & $30(5)$ & $30(6)$ & $29(6)$ & $29(6)$ \\
\hline \multirow[t]{2}{*}{ Elated - Depressed } & 0 & $27(7)$ & $27(6)$ & $27(6)$ & $27(6)$ \\
\hline & 6 & $28(7)$ & $27(6)$ & $28(6)$ & $27(7)$ \\
\hline \multirow[t]{2}{*}{ Confident - Unsure } & 0 & $24(7)$ & $25(8)$ & $25(6)$ & $24(6)$ \\
\hline & 6 & $24(8)$ & $25(7)$ & $25(7)$ & $25(7)$ \\
\hline \multirow[t]{2}{*}{ Energetic - Tired } & 0 & $25(7)$ & $25(8)$ & $25(7)$ & $24(7)$ \\
\hline & 6 & $24(8)$ & $24(8)$ & $24(8)$ & $24(7)$ \\
\hline \multirow[t]{2}{*}{ Clearheaded - Confused } & 0 & $29(6)$ & $29(6)$ & $29(6)$ & $30(6)$ \\
\hline & 6 & $29(7)$ & $29(7)$ & $29(6)$ & $30(6)$ \\
\hline \multirow[t]{2}{*}{ Total mood score } & 0 & $161(34)$ & $162(36)$ & $164(32)$ & $163(29)$ \\
\hline & 6 & $163(36)$ & $161(37)$ & $162(35)$ & $162(34)$ \\
\hline
\end{tabular}


Table 2. SF36 Health Concept scores at baseline and six months by tablet dose. Higher scores indicate a better quality of life. Data are medians and interquartile ranges. P-values refer to the interaction between time of visit and intervention dose.

\section{Intervention dose}

\begin{tabular}{|c|c|c|c|c|c|}
\hline & & & & & \\
\hline$M$ & & & & & \\
\hline Heal & & $\mathrm{n}=100$ & $\mathrm{n}=96$ & $\mathrm{n}=105$ & $\mathrm{n}=97$ \\
\hline Physical Functioning & 0 & $90(75-95)$ & $85(70-95)$ & $90(75-95)$ & $90(75-95)$ \\
\hline$(p=0.97)$ & 6 & $89(76-95)$ & $85(65-95)$ & $85(75-95)$ & $85(75-95)$ \\
\hline Role - Physical & 0 & $94(77-100)$ & $100(75-100)$ & $94(78-100)$ & $100(75-100)$ \\
\hline$(p=0.87)$ & 6 & $94(75-100)$ & $94(63-100)$ & $94(75-100)$ & $94(75-100)$ \\
\hline Role - Emotional & 0 & $100(92-100)$ & $100(100-100)$ & $100(100-100)$ & $100(100-100)$ \\
\hline$(p=0.90)$ & 6 & $100(92-100)$ & $100(100-100)$ & $100(100-100)$ & $100(100-100)$ \\
\hline Bodily Pain & 0 & $79(62-100)$ & $74(54-100)$ & $84(62-100)$ & $84(62-100)$ \\
\hline$(p=0.98)$ & 6 & $84(61-100)$ & $77(51-100)$ & $74(62-100)$ & $84(62-100)$ \\
\hline General Health & 0 & $77(62-87)$ & $72(62-87)$ & $77(67-87)$ & $75(62-87)$ \\
\hline$(p=0.94)$ & 6 & $77(63-86)$ & $72(61-84)$ & $77(67-87)$ & $72(67-86)$ \\
\hline Vitality & 0 & $69(59-75)$ & $69(50-81)$ & $75(60-81)$ & $69(56-75)$ \\
\hline$(p=0.94)$ & 6 & $69(50-81)$ & $75(56-81)$ & $75(56-81)$ & $69(56-81)$ \\
\hline Social Functioning & 0 & $100(88-100)$ & $100(88-100)$ & $100(100-100)$ & $100(100-100)$ \\
\hline$(p=0.98)$ & 6 & $100(88-100)$ & $100(78-100)$ & $100(100-100)$ & $100(100-100)$ \\
\hline Mental health & 0 & $85(75-90)$ & $85(70-90)$ & $90(80-95)$ & $85(75-90)$ \\
\hline$(p=1.0)$ & 6 & $85(75-90)$ & $85(70-90)$ & $90(75-92)$ & $85(75-90)$ \\
\hline Health transition & 0 & $50(50-50)$ & $50(50-50)$ & $50(50-50)$ & $50(50-50)$ \\
\hline$(p=0.85)$ & 6 & $50(50-50)$ & $50(50-50)$ & $50(50-50)$ & $50(50-50)$ \\
\hline
\end{tabular}


Table 3: ANCOVA summary output. In each total mood score at follow-up is the dependent variable and baseline mood the covariate. Baseline mood is the only significant predictor of six month mood. Effect sizes are presented as partial eta-squared $\left(\eta_{\mathrm{p}}{ }^{2}\right)$.

(i) Intervention dose

\begin{tabular}{lrrrrc} 
& d.f. & MS & $\mathrm{F}$ & $\mathrm{p}$ & $\eta_{\mathrm{p}}{ }^{2}$ \\
\hline Baseline mood & 1 & 264603 & 401 & $<0.001$ & 0.475 \\
Dose $(0,100,200,300 \mu \mathrm{g} / \mathrm{d})$ & 3 & 167 & 0.25 & 0.859 & 0.002 \\
Error & 443 & 659 & & & \\
\hline
\end{tabular}

$$
\mathrm{R}^{2}=0.475
$$

(ii) Gender

\begin{tabular}{lrrrrc} 
& d.f. & MS & F & p & $\eta_{\mathrm{p}}{ }^{2}$ \\
\hline Baseline mood & 1 & $2.3 \times 10^{10}$ & 392 & $<0.001$ & 0.468 \\
Gender & 1 & $9.9 \times 10^{6}$ & 0.17 & 0.682 & $<0.001$ \\
Error & 445 & $5.9 \times 10^{7}$ & & & \\
\hline
\end{tabular}

$\mathrm{R}^{2}=0.487$

(iii) Age group

\begin{tabular}{lrrrrc} 
& d.f. & MS & $\mathrm{F}$ & $\mathrm{p}$ & $\eta_{\mathrm{p}}{ }^{2}$ \\
\hline Baseline mood & 1 & $2.5 \times 10^{10}$ & 419 & $<0.001$ & 0.486 \\
Age group (60-64, 65-69, 70-74) & 2 & $1.7 \times 10^{7}$ & 0.30 & 0.745 & 0.001 \\
Error & 444 & $5.9 \times 10^{7}$ & & & \\
\hline
\end{tabular}

$\mathrm{R}^{2}=0.488$

(iv) GP practice location

\begin{tabular}{lrrrrc} 
& d.f. & MS & F & p & $\eta_{\mathrm{p}}{ }^{2}$ \\
\hline Baseline mood & 1 & $2.5 \times 10^{10}$ & 426 & $<0.001$ & 0.490 \\
Practice location & 3 & $7.4 \times 10^{7}$ & 1.25 & 0.291 & 0.008 \\
Error & 443 & $5.9 \times 10^{7}$ & & & \\
\hline
\end{tabular}

$\mathrm{R}^{2}=0.492$ 
Figure 1: Participant flow through the study

Figure 2: Selenium status was significantly altered by supplementation. Data are means and 95\% CIs at baseline (-) and six month follow-up (-) for the four intervention doses. Plasma selenium concentration can be converted to $\mu \mathrm{g} / \mathrm{L}$ by multiplying by 1.027 , the density of plasma.

Figure 3: POMS-BI total mood scores at baseline and follow-up (6 months) for participants

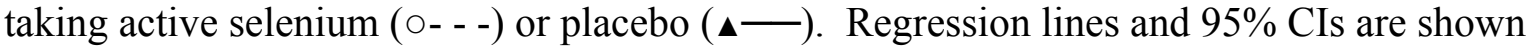
for illustration purposes only as actual analyses were performed on square-transformed data. 